Whyte, W., Green, G., and Albisu, A. (2007) Collection efficiency and design of microbial air samplers. Journal of Aerosol Science, 38 (1). pp. 97-110. ISSN 0021-8502

Copyright (C) 2006 Elsevier Ltd.

A copy can be downloaded for personal non-commercial research or study, without prior permission or charge

Content must not be changed in any way or reproduced in any format or medium without the formal permission of the copyright holder(s)

When referring to this work, full bibliographic details must be given

http://eprints.gla.ac.uk/84358

Deposited on: 20 May 2014

Enlighten - Research publications by members of the University of Glasgow http://eprints.gla.ac.uk 


\title{
Collection efficiency and design of microbial air samplers
}

\author{
W Whyte, G Green and A Albisu, \\ Department of Mechanical Engineering, University of Glasgow, Scotland, UK.
}

\begin{abstract}
The variables affecting the physical collection efficiency of air samplers of the type that impact microbecarrying particles onto agar were investigated using a simplified analytical method and computational fluid dynamics. The results from these two techniques were compared, as were the effect of jet velocity, nozzle size, and nozzle distance from the agar surface; also considered was the optimisation of these variables to obtain an efficient design of sampler. A technique is described that calculates the proportion of microbecarrying particles that a sampler will collect from a typical size distribution of microbe-carrying particles found in occupied rooms; the three air samplers studied were found to collect from about $22 \%$ to over $99 \%$ of the micro-organisms in the room air.
\end{abstract}

Keywords: Air sampler; Microbial; Impactor; Collection efficiency

\section{Introduction}

Most microbial air samplers used in occupied rooms, such as hospitals and cleanrooms, draw air containing microbe-carrying particles and accelerate it through a hole, slit, or a fan blade, and direct it towards a nutrient agar surface. As the air turns away from the agar surface the microbe-carrying particles that cannot follow the flow are impacted. The plate containing nutrient agar is then incubated at a suitable temperature and time, and the resulting microbial colonies are counted to ascertain the number of microbecontaining particles collected from a given volume of air.

Ljungqvist and Reinmuller (1998) compared a selection of air samplers currently used in rooms and demonstrated that some samplers collect up to 10 times less microbes than others, thus showing that when a low efficiency sampler is used, unhygienic rooms can incorrectly appear to achieve the correct cleanliness standard. To design a microbial air sampler to achieve a high sampling efficiency, consideration should be given to particle losses around the intake to the sampler but the most important problem is inefficient impaction of the smaller sizes of microbe-carrying particles on the agar surface, usually because there is too low an impaction velocity.

The first microbial air samplers were produced in the 1940s and 1950s and designed to efficiently impact single-cell microbes with a diameter of about 1-2 $\mu \mathrm{m}$. This size was chosen, as air samplers were often designed by investigators working in the field of microbial warfare, where microbes were generated as an aerosol of single cells with a diameter of about 1-2 $\mu \mathrm{m}$. However, microbial air samplers are extensively used in hospitals and cleanrooms, where the requirement is for samplers that are quieter, smaller, and more portable than these earlier designs. As a consequence, modern samplers often have smaller less-powerful air pumps and lower impact velocity. It is not clear what effect this has had on the collection of microbecarrying particles found in occupied rooms, although Möller (2002) considers that some of the samplers designed in the last decade or two, have too low an impaction velocity to efficiently collect a high percentage of microbe-carrying particles. When the early samplers were designed, it was not realised that the majority of microbes in an occupied room are carried on skin cells, or fragments of skin cells, dispersed by people in the room. This was not known until the 1960s (Davies and Noble, 1962), when it was also found microbecarrying particles had an average equivalent diameter of about $12 \mu \mathrm{m}$ (Noble, Lidwell and Kingston, 1963). Microbe-carrying particles with an average size of $12 \mu \mathrm{m}$ are easier to impact than unicellular microorganisms of $1-2 \mu \mathrm{m}$ in size. It is therefore possible that microbial samplers with lower collection efficiencies 
than early samplers may still collect a large proportion of the microbe-carrying particles of the sizes found in an occupied room.

The collection efficiency of microbial air sampler can be considered in two ways:

- physical collection efficiency,

- biological collection efficiency.

The physical collection efficiency measures the collection efficiency of inert particles. The biological efficiency measures the physical efficiency, but also the losses in viability of micro-organisms during sampling that are caused by cell damage or stress, through impaction or dehydration. The effect of impact damage and stress on microbes over a range of velocities from 24 to $250 \mathrm{~m} / \mathrm{s}$ was studied by Stewart et al (1995) who found that the highest rate of recovery of viable cells of Pseudomonas fluorescens was achieved at a velocity of $40 \mathrm{~m} / \mathrm{s}$, and for Micrococcus luteus it was $24 \mathrm{~m} / \mathrm{s}$. They investigated metabolic and structural damage, and found the Gram-positive micro-organism more robust than the Gram-negative one. Their conclusions different from May and Harper (1957) who studied a liquid impinger sampler and found that the loss in the viability of Serratia marcescens, which was selected because of its known high sensitivity to collection, was around $20 \%$ when impacted at near to sonic velocity $(340 \mathrm{~m} / \mathrm{s})$. When the air velocity was reduced to about $130 \mathrm{~m} / \mathrm{s}$ the losses were reduced to almost zero.

Information on how to design impactors has been reported by several authors, such as Hinds (1999), Nevalainen, Pastuszka, Liebhaber and Willeke (1992), and Marple and Willeke (1976). Marple and Willeke (1976) based their design method on a study carried out by Marple and Liu (1974), who used computational fluid dynamics (CFD) to calculate the efficiency of particle impaction, the accuracy of the method being confirmed experimentally. Marple and Willeke (1976) suggest the following method:

1. Ensure that the Reynolds number of the air passing through the nozzle is between 500 and 3000

2. Select an appropriate $d_{50}$ 'cut-off' size of particle, and calculate and select the variables required to achieve this cut off size. Marples and Willeke’s method uses Stokes number, but their method is basically the same as the 'simplified analytical method' employed in this paper.

3. Ensure that the ratio of the separation distance (S) (known in this paper as the nozzle-to surface distance ${ }^{1}$ ) to the diameter or width of the nozzle (W) is not less than 1.5 in rectangular nozzles and 1 in round nozzles. This ratio is given by a dimensionless number that is generally known as the $S / W$ ratio.

4. If possible, the entrance to the nozzle should be tapered or conical, and of constant width or diameter, with a nozzle throat length at least as large as the width or diameter of the nozzle.

Marple and Willeke's method is widely used, and it is therefore appropriate that the results and conclusions in this published paper should be compared with their recommendations and, in particular, their $S / W$ requirements.

Note $^{1}$ : In this paper we have avoided giving the meaning of the word 'jet' to a nozzle, but use 'jet' as a description of the fluid coming from a nozzle. Also, in air samplers, plates containing nutrient agar are used to collect the microbe-carrying particles impacted onto the agar surface. In this situation it is best not to call the agar surface the 'plate'. We therefore use the term 'nozzle-to-surface' rather than the commonly used 'jet-to-plate'.

\section{Simplified analytical approach for calculating collection efficiency}

A method used to calculate the collection efficiency of samplers of the impactor type was established many years ago by May (1945); Ranz and Wong, (1950) and Davies and Aylward (1951). This is called in this paper the 'simplified analytical method'. Shown in Fig. 1 is an intake orifice of an air sampler, with only one streamline considered. The air passes through the intake at a velocity $\mathbf{U}$, and as it approaches the agar surface it turns, the arc of the turning circle being known in this paper as the 'radius of curvature', which is assumed to be the same as the radius of the intake nozzle, or half the width of the slit. The velocity round the curve is assumed to be $\mathbf{U}$. 


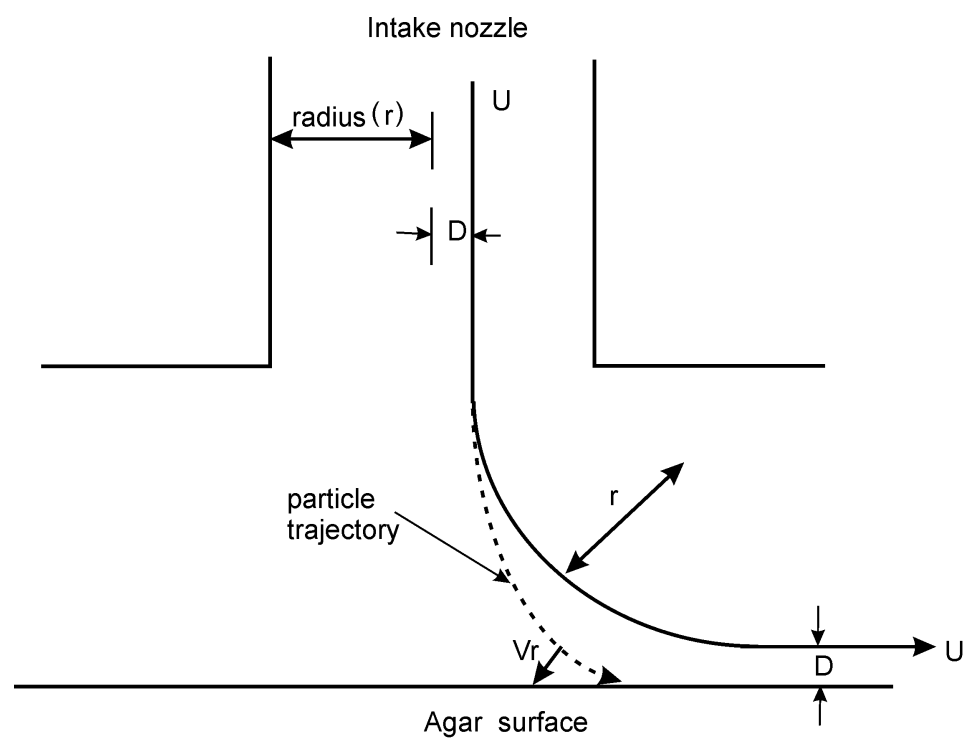

Fig. 1. Impaction of a particle on a surface after exiting a nozzle

A particle travelling along the streamline experiences a centrifugal force that causes the particle to move toward the nutrient agar surface. The collection efficiency (E) is given in Eq. (1), the derivation of this equation being given by Hinds (1999).

$$
E=\frac{\pi \cdot U}{2 \cdot r}\left(\frac{\rho \cdot d^{2} \cdot C}{18 \eta}\right)
$$

where, $\mathbf{U}$ is the velocity of the air through the nozzle, $\boldsymbol{r}$ is the radius of curvature of the streamline, $\boldsymbol{\rho}$ is the density of microbe-carrying particles $\left(1100 \mathrm{~kg} / \mathrm{m}^{3}\right), \boldsymbol{d}$ is the equivalent particle diameter, $\boldsymbol{C}$ is the Cunningham slip factor, and, $\boldsymbol{\eta}$ is the viscosity of room air at $20^{\circ} \mathrm{C}\left(1.81 \times 10^{-5} \mathrm{~Pa} . \mathrm{s}\right)$.

The collection efficiency of a sampler over a range of particle sizes can be calculated from Eq. (1), and then the $d_{50}$ 'cut off' size. The $d_{50}$ size is often used to describe the impact efficiency of a sampler, it being the particle size at which $50 \%$ of the particles are collected, and $50 \%$ pass through the sampler because that are too small to impact.

\section{Computational fluid dynamics (CFD)}

The simplified analytical method described in the previous section has the following simplifying assumptions:

1. The distance between the nozzle outlet and collection surface is not considered, it being assumed that the radius of curvature of the air stream passing the agar surface remains constant, irrespective of the nozzleto-surface distance, and is equal to the nozzle radius or half the slit width;

2. the radius of curvature remains constant, irrespective of the air velocity;

3. the velocity passing the agar surface is the same as the nozzle velocity.

To avoid relying on these assumptions, and obtain more accurate results, the path and velocities of the streamlines within a given geometry of a sampler are determined so that the trajectories and velocities of different sizes of particles, and the efficiency of impaction, can be calculated (Marples and Liu, 1974). This can be done using commercially available CFD software, and Fluent flow-modelling software, available from Fluent Inc., was used in this investigation.

When using CFD analysis, a number of decisions have to be made. Because the particle trajectories are required, the Euler-Lagrange method was used, as was the stochastic tracking approach when modelling the dispersion of particles due to turbulence. The likely nature of the air flow i.e. laminar or turbulent can be 
determined by calculation of the Reynolds number. The Reynolds number of the air passing through the intake nozzles of most microbial air samplers used in occupied rooms is likely to fall within the range of 500 to 1500. The samplers studied in this paper, and described later, are known as the MG, SAS and SMA samplers and had Reynolds numbers of the air passing through the nozzles of 1425, 1158 and 525, respectively, the airflow therefore being assumed to be laminar. However, when the air passes out of the nozzle it expands within the sampler's passages and the characteristic dimension increases and hence the Reynolds number. Because of the geometry of the samplers and possible columnar support of adjacent jets it is often not clear what characteristic dimension should be used. The air jet is also directed to the agar surface facing it, which may also cause the airflow to become more turbulent. The airflow passing the agar surface may therefore be turbulent in some samplers and laminar in others. Because of this, the impact efficiencies over a range of particle sizes were calculated using both laminar and turbulent flow models. Both models gave similar results, but the cut-off with the turbulence model was not as sharp; this was as expected.

When turbulent flow is investigated, the best choice of a model to simulate an expanding jet of air directed at a surface has to be determined. A number of turbulent flow models available in Fluent were inappropriate, the most suitable being the $\mathrm{K}-\varepsilon$ and $\mathrm{K}-\omega$ models. A comparison was made between the results from these models and a sampler whose experimental collection efficiency over a size range of microbe-carrying particles was published by Lach (1985). The K- $\omega$ standard method did not give similar results but all of the K- $\varepsilon$ models were reasonably close to the experimental results, with the K- $\varepsilon$ 'realizable model with standard wall function' slightly better than the others, and this was chosen.

For reasons discussed later, the impact efficiency of particles of a diameter between 0.5 and $50 \mu \mathrm{m}$ were studied. To obtain the trajectories of these particles, the airflow field was obtained and a release was simulated of particles of a density of $1100 \mathrm{~kg} / \mathrm{m}^{3}$ (the density of skin particles reported by Shi and X, 2004) that were evenly distributed across the intake nozzle. The impaction of particles was ascertained and the impact efficiency calculated. As nutrient agar is more than $95 \%$ water, all the impacted particles were assumed to be retained, and not to 'bounce off', as can occur with solid surfaces.

The impact efficiency of samplers with rectangular slits is reported to be less efficient than samplers with round nozzles but the use of the 'hydraulic' diameter minimises this difference (Willeke and McFeters, 1975). The hydraulic diameter was therefore used as the characteristic dimension of the intake nozzles. This is equal to 4 times the cross section area of the nozzle divided by its wetted perimeter length; in the case of a round nozzle it is the same as the actual diameter.

\section{CFD investigation of a validated sampler}

Lach (1985) reported an experimental study into the collection efficiency of a range of sizes of microbecarrying particles impacted by a SAS sampler. Lach studied a version of the sampler older than the current model, but the current version has the same physical characteristics that influence collection efficiency. These are given in Table 1 . The sampler's air volume was reported by Lach to be $180 \mathrm{l} / \mathrm{min}$, but discussions with the manufacturer revealed that independent testing laboratories have checked the sampling volume, and the volume that Lach should have used was $200 \mathrm{l} / \mathrm{min}$; this value was used to calculate the velocity through the nozzles.

Table 1. Characteristics of the SAS air sampler

\begin{tabular}{ll}
\hline Number of intake nozzles & 220 \\
Nozzle radius (mm) & 0.5 \\
Hydraulic diameter & 1 \\
Nozzle-to-agar surface distance (mm) & 2 \\
Nozzle velocity (m/s) & 17.4 \\
S/W ratio & 2 \\
\hline
\end{tabular}

Shown in Fig. 2 is the simulation of the trajectories of 10 particles of $1 \mu \mathrm{m}$ diameter in a SAS sampler. Only ten particles were chosen to clearly show the trajectories but in a typical CFD analysis the release of 
many more particles was simulated. The intake nozzle and air passages of the microbial air samplers studied are symmetrical around the nozzle intake and therefore only one half the area of the intake and outlet passages are shown. As $1 \mu \mathrm{m}$ particles have a low inertia, the trajectories are very similar to the air streamlines. It may be seen that the air jet from the nozzle travels down to the agar surface where it turns and moves out by the outlet passage. An anticlockwise air vortex is induced by the jet in the upper two thirds of the outlet passage close to the inlet nozzle.

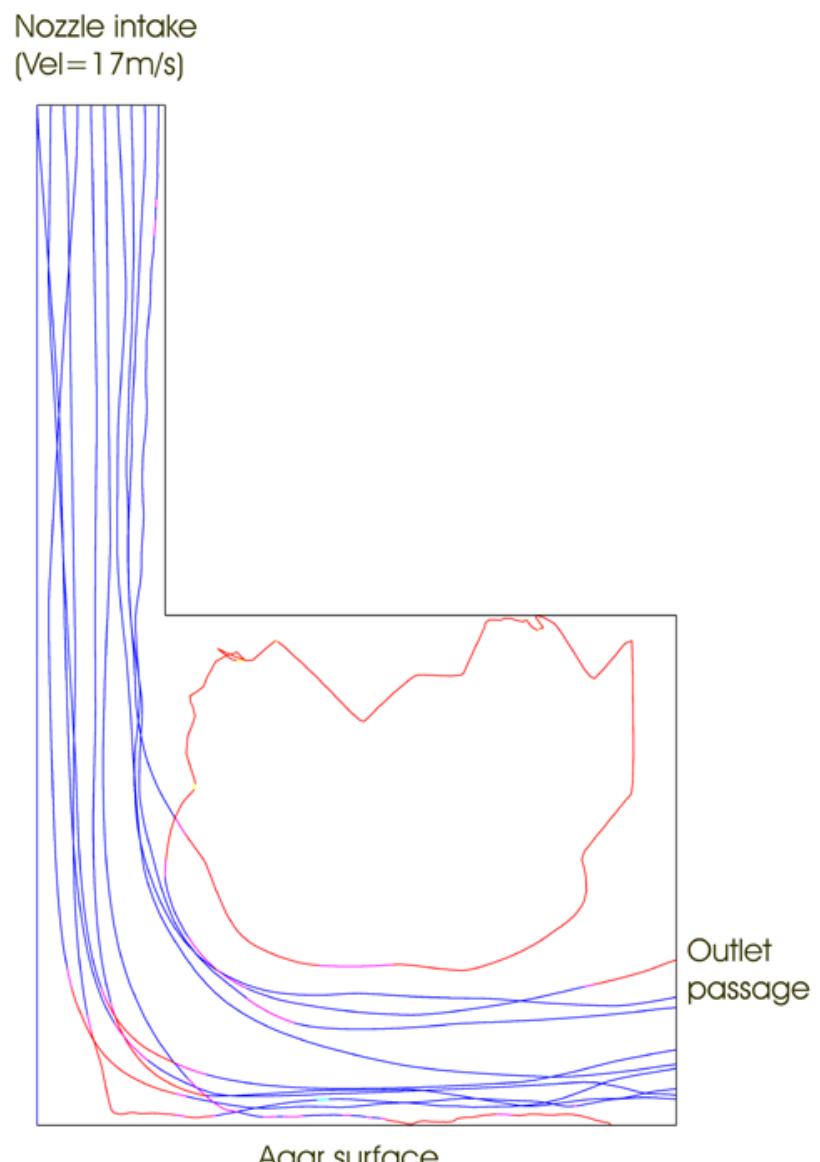

Fig. 2. Trajectories of $1 \mu \mathrm{m}$ particles in the SAS sampler

Shown in Fig. 3 is a graph of the impaction efficiencies over a range of particle sizes for the SAS sampler when calculated by both the CFD and simplified analytical analysis, and the experimental results obtained by Lach (1985).

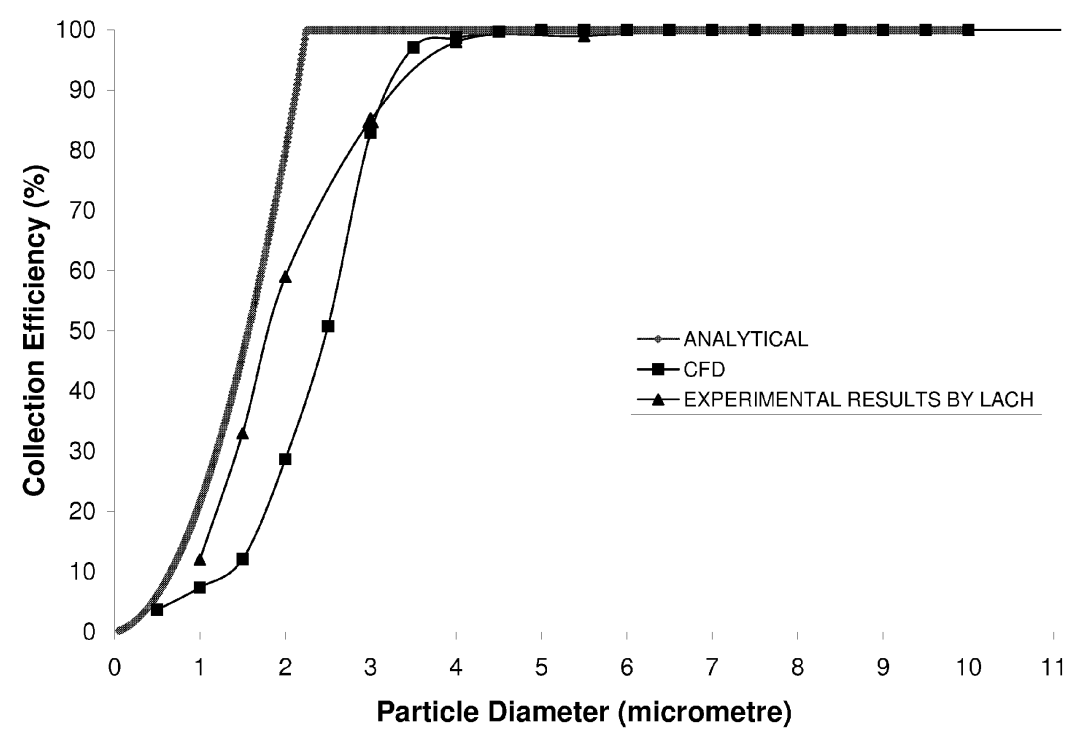

Fig. 3. Collection efficiency of the SAS sampler 


\section{Simplified analytical approach compared to a CFD approach}

Further investigations of impact efficiency were made of two further designs of popular samplers, with widely different properties of the type that influences collection efficiency i.e. nozzle size and air velocity. These were the Mattson Garvin (MG) sampler (the Air Trace sampler has the same nozzle size and air velocity) and SMA Atrium samplers. Within the range of currently-available samplers, the MG sampler has a fine slit width and high velocity, the SMA had a large diameter nozzle and low velocity, and the SAS had moderate nozzle diameter and velocity. The relevant standard dimensions of these samplers are given in Table 2. When using the CFD analysis, the model adopted for the MG and SAS samplers was turbulent, and for the SMA sampler it was laminar.

Table 2. Physical characteristics of additional air samplers studied

\begin{tabular}{lll}
\hline & MG & SMA \\
\hline Number of intake nozzles & 1 slit & 12 holes \\
Nozzle radius, or half slit size (mm) & 0.076 & 3.17 \\
Hydraulic diameter & 0.303 & 6.35 \\
Nozzle-to-plate distance (mm) & 2.5 & 4 \\
Nozzle velocity (m/s) & 70.5 & 1.2 \\
$S / W$ ratio & 16 & 1.6 \\
\hline
\end{tabular}

Table 3 gives a comparison of the $d_{50}$ particle sizes of the three samplers obtained from the CFD and simplified analytical methods, and in the case of the SAS sampler, to the experimental results. The reasons for the similarities and differences of the results obtained from the two methods of analysis were further explored.

Table 3. $d_{50}$ sizes obtained by simplified analytical and CFD approaches

\begin{tabular}{llll}
\hline & Simplified analytical $(\mu \mathrm{m})$ & CFD $(\mu \mathrm{m})$ & Experimental result $(\mu \mathrm{m})$ \\
\hline MG & 0.25 & 0.23 & - \\
SAS & 1.57 & 2.48 & 1.85 \\
SMA & 15.5 & 18.6 & - \\
\hline
\end{tabular}

\section{Investigations into collection efficiency of microbial air samplers}

In the simplified analytical method, it is considered that the radius of curvature of the air streamlines as they pass the agar surface is best characterised by the nozzle radius, or half the width of a rectangular nozzle. This will be achieved when the $S / W$ ratio is about 2 . However, if the nozzle-to-surface distance changes, the flow field and the radius of curvature will change, and hence the efficiency of impaction. As the nozzle-to-surface distance decreases, the closing of the two surfaces will force a change in the flow field and reduction in the radius of curvature. When the nozzle-to-surface distance increases, the air jet will expand and diffuse, but the rate of change of the flow field and radius of curvature will not be as great. Marples and Willeke (1976) suggested that the $S / W$ ratio should not be below 1 in round nozzles and 1.5 in rectangular nozzles, or the $d_{50}$ particle size will deviate from the size calculated. They considered large $S / W$ ratios unimportant. However, there must be a point where the $S / W$ ratio becomes large enough to influence the radius of curvature, and reduce the efficiency of impaction. Hinds (1999) suggested that the S/W ratio should not exceed 5.

If a microbial air sampler has a fine nozzle size, then the $S / W$ ratio may be larger than 5 e.g. the MG sampler has a $S / W$ ratio of 16 . Samplers with a large nozzle size e.g. the SMA sampler have a $S / W$ ratio below 1 . The effect of nozzle-to-agar distances, and their associated $S / W$ ratios, on impact efficiency was therefore studied. Also studied was impact velocity.

Some types of microbial samplers use electro-mechanical means to maintain the surface of the agar plate at a constant distance from the nozzle. Other samplers maintain the distance by using an agar plate with a 
constant and known amount of agar in them. However, in the latter method, owing to the design distance, variations in agar thickness across the plate, evaporation of water during storage, and mistakes in the amount poured, the nozzle-to-surface distances may vary from 1 to $10 \mathrm{~mm}$, although values close to 1 or $10 \mathrm{~mm}$ would be considered, in the practical sense, extreme values. This was the range of distances studied.

\subsection{The effect of the nozzle-to-agar distances}

The airflow within a SAS sampler has been previously discussed, and shown in Fig. 2. Shown in Table 4 is the $d_{50}$ values obtained by the CFD method, when the nozzle-to-surface distance (and $S / W$ ratio) was varied from 0.5 to 10 , and the air velocity and nozzle size kept constant at the standard values. These results show that over the range of $S / W$ values from 2 to 10, the SAS sampler has a fairly constant $d_{50}$ of between 2.5 and $2.2 \mu \mathrm{m}$, but drops to $1.5 \mu \mathrm{m}$ when the nozzle-to-surface distance (and $S / W$ ratio) is reduced to 0.5 . This would be in agreement with the suggestion that the $S / W$ ratio should be kept between 1 and 5 , although a $S / W$ ratio of 10 has little effect on the $d_{50}$ size.

Table 4. Effect of nozzle-to-surface distances, and $S / W$ ratio, on the $d_{50}$ collection sizes of the SAS

\begin{tabular}{lllll}
\hline Nozzle-to-surface distance $(\mathrm{mm})$ & 0.5 & 2 & 6 & 10 \\
\hline$S / W$ ratio & 0.5 & 2 & 6 & 10 \\
$d_{50}$ collection size $(\mu \mathrm{m})$ & 1.5 & 2.5 & 2.2 & 2.1 \\
\hline
\end{tabular}

Shown in Fig. 4 is the airflow field within the SMA sampler, as determined by CFD analysis, when the nozzle-to-surface distance was kept constant at the standard value of $4 \mathrm{~mm}(S / W$ ratio $=0.63)$. The SAS sampler has several large nozzles of $6.34 \mathrm{~mm}$ through which air passes at a low velocity of $1.2 \mathrm{~m} / \mathrm{s}$. As a consequence, in comparison to the SAS sampler, the air jet from the nozzle does not flow so strongly and compactly to the agar surface, but moves out of the sampler's passages more directly, the air expanding to fill a larger proportion of the outlet passage close to the nozzle, with only about one-third of the upper passage being filled with an anticlockwise air vortex. Inspection of the airflow fields of the SMA sampler (not reproduced here) shows that as the nozzle-to-surface distance changes so does the radius of curvature of the air passing the agar surface. The consequence of this is shown in Table 5. When the nozzle-to-surface is $1 \mathrm{~mm}$ distance $(S / W$ ratio $=0.16)$ the $\mathrm{d}_{50}$ is $7.3 \mu \mathrm{m}$ but, as the nozzle-to-surface distance increases, the impact efficiency decreases, until at $10 \mathrm{~mm}$ distance $(\mathrm{S} / \mathrm{W}$ ratio $=1.6)$ it is $30 \mu \mathrm{m}$. According to Marples and Willeke's (1976), the $d_{50}$ cut-off size, as calculated by the simple analytical method $(15.5 \mu \mathrm{m})$, should remain constant over a range of $S / W$ ratios greater than 1 . It can be seen in the case of the SMA sampler that the $\mathrm{d}_{50}$ cut-off sizes varies when the S/W ratio is less than 1 , but also when the $S / W$ ratio is above 1 . It should also be noted that at the standard nozzle-to-surface distance of $4 \mathrm{~mm}$ was similar to the nozzle radius (3.2 $\mathrm{mm}$ ) of the sampler, and hence the $d_{50}$ values calculated by the CFD and simplified analytical method at that dimension are very similar. Therefore, when a comparison is made between the $d_{50}$ cut-off size obtained by the simplified analytical and CFD methods (as in Table 3), it should be realised that in the SMA sampler the $d_{50}$ size obtained by the CFD method is strongly influenced by the nozzle-to-distance chosen.

Table 5. Effect of nozzle-to-surface distances, and S/W ratio, on the $d_{50}$ collection size of the SMA

\begin{tabular}{llllll}
\hline Nozzle-to surface distance $(\mathrm{mm})$ & 1 & 2 & 4 & 6 & 10 \\
\hline$d_{50}(\mu \mathrm{m})$ & 7.3 & 13.1 & 18.6 & 23.2 & 29.7 \\
$S / W$ ratio & 0.16 & 0.32 & 0.63 & 0.95 & 1.6 \\
\hline
\end{tabular}




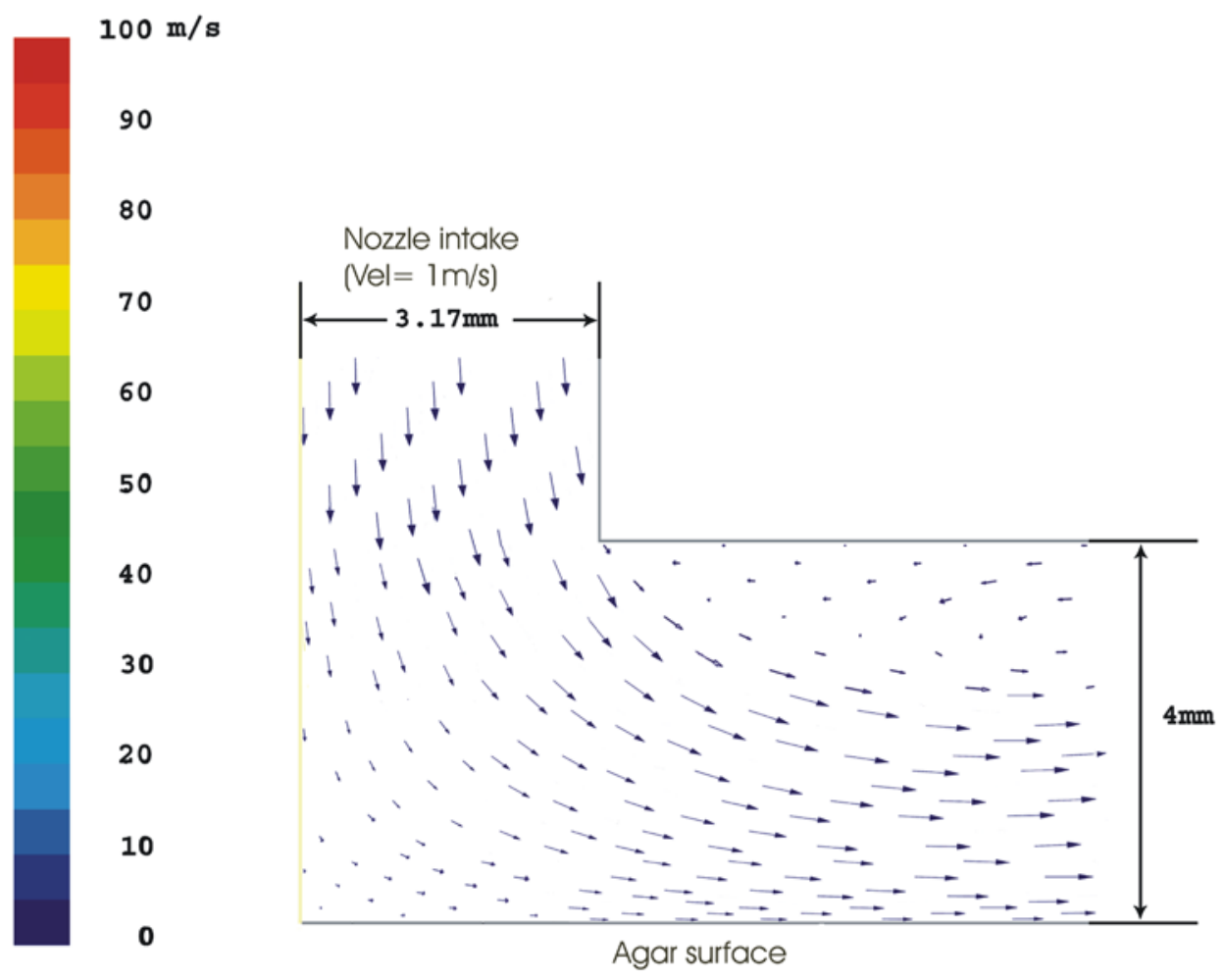

Fig. 4 Air flowing from the nozzle in the SMA sampler

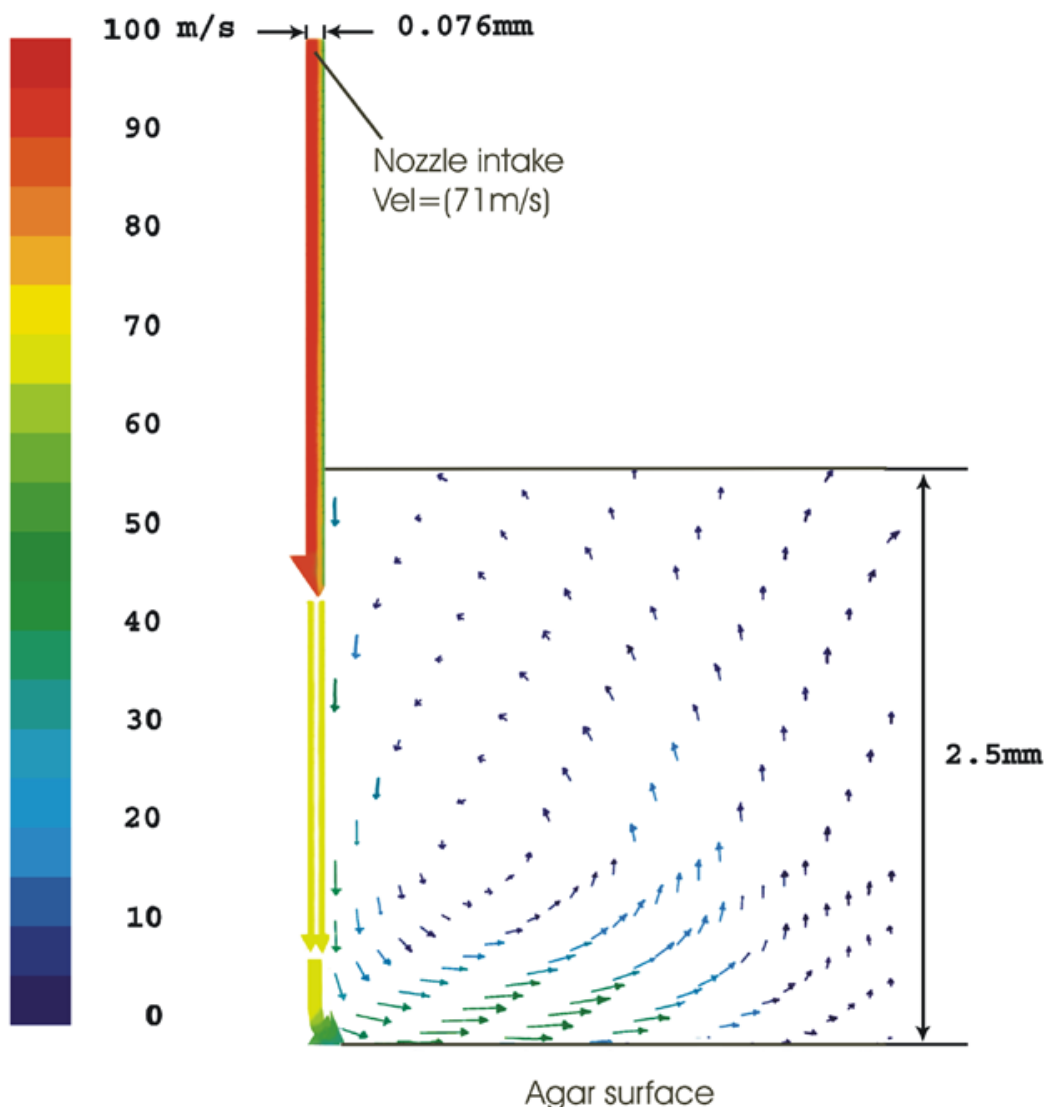

Fig. 5 Air flowing from the nozzle in the MG sampler 
The flow field of the MG sampler, with the standard slit width and nozzle-to-surface distances ( $S / W$ ratio of 16), is given in Figure 5. It shows that the MG sampler, with its narrow slit and high air velocity, retains a compact jet of air down to the agar surface and, as it turns, the radius of curvature is similar to its intake radius. It also has a vortex induced over the upper three quarters of the outlet passage. The effect of different nozzle-to-surface distances (and $S / W$ ratios) on the $d_{50}$ sizes was not calculated, but further information on the MG sampler was gathered by studying the effect of air velocity and nozzle size.

\subsection{Effect of air velocity and nozzle size on impact efficiency}

The effect of air velocity on the impact efficiency was studied in the MG sampler using both CFD and simplified analytical methods, and the results are shown in Table 6. The standard half-slit width of $0.076 \mathrm{~mm}$ and nozzle-to-surface distance of $2.5 \mathrm{~mm}$ (giving an $S / W$ value of 16 ) were kept constant. As expected, it was found that an increase in velocity was accompanied by an increase in impact efficiency. The $d_{50}$ calculated by the simple analytical method was found to be close to that calculated by the CFD method, although there is a tendency at lower and higher velocities for the values to diverge, presumably as the CFD method predicts a different radius of curvature than assumed in the simple analytical method.

Table 6. $\mathrm{d}_{50}$ for different velocities in the MG sampler $(S / W=16.4)$

\begin{tabular}{llllll}
\hline Velocity $(\mathrm{m} / \mathrm{s})$ & 20 & 30 & 50 & 70 & 90 \\
\hline$d_{50}$-calculated by CFD & 0.8 & 0.58 & 0.33 & 0.25 & 0.17 \\
$d_{50}$-calculated by simplified analytical method & 0.52 & 0.41 & 0.30 & 0.25 & 0.21 \\
\hline
\end{tabular}

Table 7. $d_{50}$ in a MG sampler for different nozzle widths and velocity of $70 \mathrm{~m} / \mathrm{s}(S / W=16.4)$.

\begin{tabular}{lccccc}
\hline Half slit size $(\mathrm{mm})$ & 0.076 & 0.1 & 0.5 & 0.7 & 1.0 \\
\hline$d_{50}$-calculated by CFD & 0.23 & 0.26 & 0.95 & 1.29 & 1.63 \\
$d_{50}$-calculated by simplified analytical method & 0.25 & 0.29 & 0.74 & 1.08 & 2.51 \\
\hline
\end{tabular}

The effect of slit width on the impact efficiency of the MG sampler was obtained using its standard high velocity of $70 \mathrm{~m} / \mathrm{s}$ and calculated by CFD and simplified analytical methods. The results are shown in Table 7 as $d_{50}$ sizes. The two methods of calculation give a similar result, although there is a divergence at larger slit sizes where the CFD method would be expected to predict a smaller radius of curvature than that assumed in the simplified analytical method. The effect of slit width on the impact efficiency of the MG sampler was also investigated at a low velocity of $20 \mathrm{~m} / \mathrm{s}$. The results given in Table 8 show that the CFD method calculated a larger $d_{50}$ for the slit sizes studies, this presumably being because at such a velocity and large $S / W$ ratio the CFD method will predict a larger radius of curvature that assumed in the simplified analytical calculations.

Table 8. $d_{50}$ in a MG sampler for different nozzle widths and velocity of $20 \mathrm{~m} / \mathrm{s}(\mathrm{S} / \mathrm{W}=16.4)$.

\begin{tabular}{lcccc}
\hline Half slit size $(\mathrm{mm})$ & 0.1 & 0.5 & 0.7 & 1.0 \\
\hline $\mathrm{d}_{50}$-calculated by CFD $(\mu \mathrm{m})$ & 1.04 & 1.75 & 2.56 & 3.21 \\
$\mathrm{~d}_{50}-$ calculated by simplified analytical method $(\mu \mathrm{m})$ & 0.29 & 0.74 & 0.89 & 2.1 \\
\hline
\end{tabular}

ISO 14698-1 (2003) suggests that microbial air samplers should be able to sample microbe-carrying particles down to $1 \mu \mathrm{m}$. It can be seen in Table 7 that when the standard velocity of $70 \mathrm{~m} / \mathrm{s}$ is used, a $d_{50}$ of just below $1 \mu \mathrm{m}$ was obtained by a half-slit size of $0.5 \mathrm{~mm}$, and Table 8 shows that where the velocity is $20 \mathrm{~m} / \mathrm{s}$ a $d_{50}$ close to $1 \mu \mathrm{m}$ is obtained with a half-slit size of $0.1 \mathrm{~mm}$. 


\section{Overall collection efficiency of naturally occurring microbes in room air}

The information discussed above can be used to determine the impact efficiency of a sampler at a discrete size of microbe-carrying particle, or to calculate the $d_{50}$ cut-off value. However, microbe-carrying particles in the air of an occupied room exist in a variety of sizes, the smaller ones being more difficult to collect. It is important to know what proportion of the various sizes of all the microbe-carrying particles in the room air are collected by a sampler, so that a sampler's overall collection efficiency can be determined.

Microbe-carrying particles have a variety of shapes and densities that influence their movement in air and it is conventional to consider the size of airborne particles in terms of equivalent particle diameter, which is the diameter of a sphere of unit density $\left(1 \mathrm{~g} / \mathrm{cm}^{3}\right)$ that has the same aerodynamic properties as the particle in question. It is known that the majority of microbes in room air are carried on skin cells, or fragments of skin cells (Davies and Noble, 1962). The frequency of occurrence of different sizes of microbe-carrying particles in hospital rooms has been reported (Noble, Lidwell and Kingston, 1963), as has that from people wearing cleanroom clothing (Whyte, 1986). These microbes were grown aerobically on non-selective nutrient agar media and incubated at $37^{\circ} \mathrm{C}$ for 48 hours. Compiling these results it can be ascertained that the size distribution of airborne microbe-carrying particles in occupied rooms conforms well to a log-normal distribution, with an average equivalent diameter of $12 \mu \mathrm{m}$, and $25 \%$ and $75 \%$ being larger than $20 \mu \mathrm{m}$ and 4 $\mu \mathrm{m}$, respectively. Microbe-carrying particles with an equivalent particle diameter above $50 \mu \mathrm{m}$ are relatively few, these having a frequency of occurrence of less than $5 \%$. Microbes sampled in the air of cleanrooms are mainly bacteria with a small proportion of spores, these micro-organisms having a unicellular size that is unlikely to be much less than $1 \mu \mathrm{m}$, and at that size a frequency of occurrence of less than $1 \%$. The information about the probability of occurrence of microbe-carrying particles equal to and greater than given sizes i.e. the cumulative size, is given in Table 9 and plotted in Figure 6 as probability density with respect to size.

Table 9 Probability in an occupied room of the occurrence of microbe-carrying particles equal to, and greater than, a given size

\begin{tabular}{lccccc}
\hline Equivalent particle diameter $(\mu \mathrm{m})$ & $\geq 1$ & $\geq 4$ & $\geq 12$ & $\geq 20$ & $\geq 50$ \\
\hline Probability of occurrence & 0.99 & 0.75 & 0.5 & 0.25 & 0.05 \\
\hline
\end{tabular}

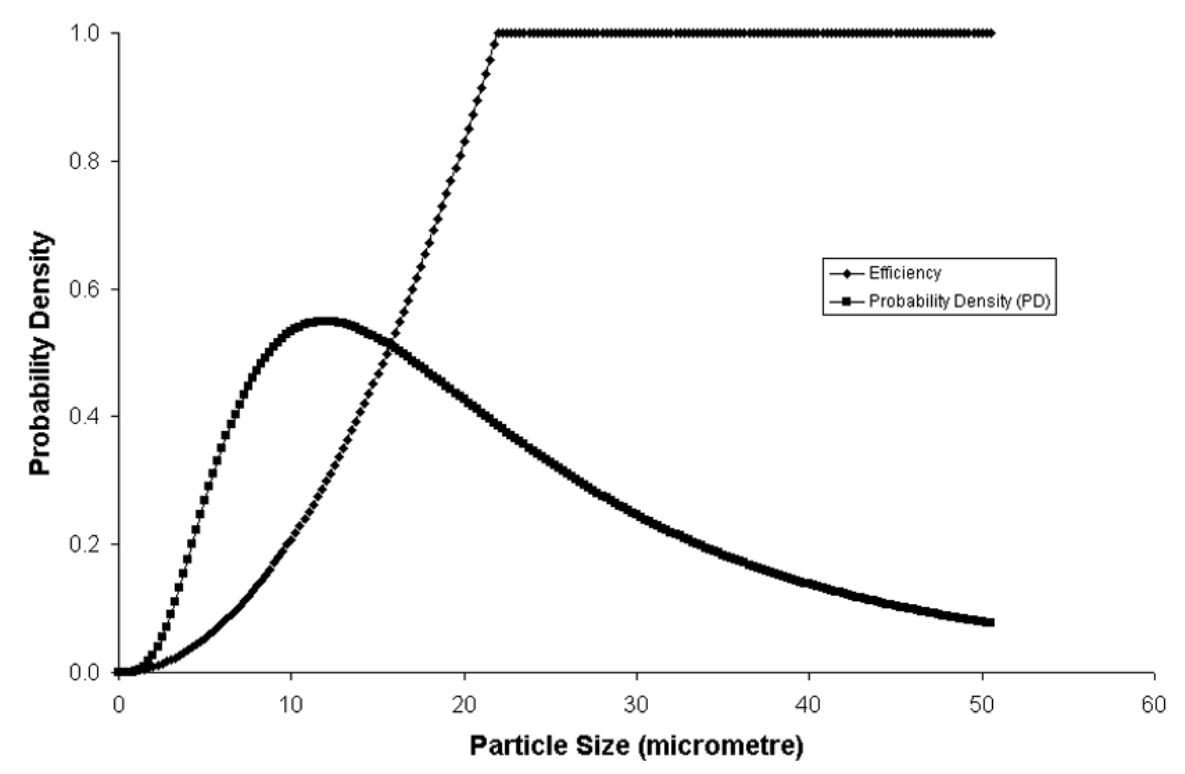

Fig. 6 The pdf of microbe carrying particles in an occupied room and the collection efficiency of the SMA sampler calculated by simplified analytical method. 
Overlaid in Figure 6 is the impact efficiency curve of the SMA sampler, as predicted by the simplified analytical method. The impact efficiency of the sampler reaches a maximum at a given particle size (approximately $22 \mu \mathrm{m}$ in this case), and all particles above that size will be collected. The probability of microbe-carrying particles at each size below $22 \mu \mathrm{m}$ being impacted onto the agar surface is dependent on the impact efficiency at each size, and this can be found by multiplying the values for each size obtained from the probability density curve with the equivalent values obtained from the impact efficiency curve. The result is a local modification of the probability density curve, as shown in Fig. 7.

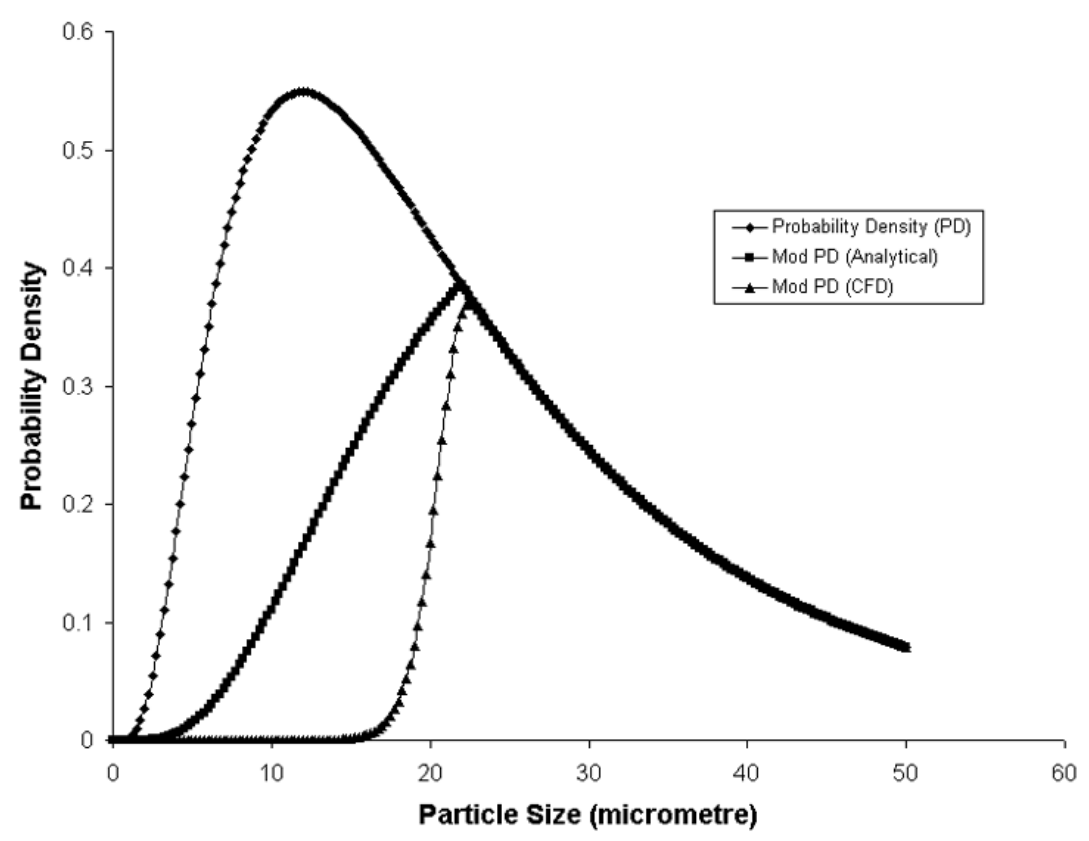

Fig. 7 The pdf of microbe carrying particles and its modification by the collection efficiency of the SMA sampler as calculated for both the simplified analytical and CFD methods.

The resultant change in the area under the modified probability curve from 0 to $22 \mu \mathrm{m}$, measures the proportion of particles not collected by the sampler. If we accept that the area under the unmodified curve, between the limits of 0 and $50 \mu \mathrm{m}$, represents almost all of the microbe-carrying particles available in room air for collection, then the area under the modified curve, between these limits, will give the proportion of microbe-carrying particles impacted with respect to the total number passing through the sampler. This information is obtained by integrating both curves between the limits of $0 \mu \mathrm{m}$ and $50 \mu \mathrm{m}$, and expressing the area under the modified curve as a percentage of the area under the original unmodified curve.

The efficiency of collection of the SMA sampler of all the microbe-carrying particles in the room air was also obtained using the CFD method. An equation was obtained by subjecting the impact efficiency results obtained by the CFD method to nonlinear least-squares fitting. The resulting equation was then used to modify the probability density curve in the same way as described for the simplified analytical method, and the proportion of collected microbe-carrying particles, compared to the total in the room air, was calculated as before. The result is shown in Figure 7. The overall collection efficiencies for all three air samplers of the range of sizes of microbe-carrying particles found in an occupied room, as calculated by the simplified analytical and CFD approaches, are given in Table 10, and can be seen to vary from about $20 \%$ to almost $100 \%$.

Table 10 Collection efficiency of three samplers of microbe-carrying particles in an occupied room

\begin{tabular}{llll}
\hline & MG & SAS & SMA \\
\hline Proportion calculated using the simplified analytical approach (\%) & 99.9999 & 99.7 & 42.55 \\
Proportion calculated using the CFD approach (\%) & 99.9994 & 98.2 & 21.8 \\
\hline
\end{tabular}




\section{Discussion and Conclusions}

This paper describes the use of simplified analytical and CFD methods to calculate the impact efficiency of microbial air samplers. The simplified analytical approach uses an equation to calculate the impact efficiency of different sizes of airborne particles, and assumes that the radius of curvature of the air streamlines as they pass the agar surface to be equal to the nozzle radius or half the slit width, this distance remaining constant irrespective of the nozzle-to-surface distance, and not affected by velocity. The CFD method does not make such an assumption but calculates the actual flow field within a sampler and hence the amount of impaction. However, using CFD is a time consuming method and requires expert knowledge of the software. It was therefore one of the objectives of this paper to find out if the simplified analytical approach was sufficiently accurate to design microbial samplers.

For similar results to be obtained from the simplified analytical and CFD methods, the radius of curvature of the air passing the agar surface should equal the radius of the nozzle, or half width of the slit, This is likely if the $S / W$ ratio is kept between 1 and 5 . However, microbial samplers commonly used in occupied rooms have $S / W$ ratios outwith this range. Our results also show that the air velocity affects the likelihood of obtaining similar results. With low velocities, as found in the SMA sampler, the $d_{50}$ cut-off size was found to change rapidly across the range of $S / W$ ratios, including those between 1 and 5. This was presumably because, at a low velocity, the flow field and the radius of curvature, is more easily changed than at high velocity. If a good estimate of impaction efficiency in this type of sampler is required, CFD analysis would be necessary.

Samplers with a moderate velocity and slit width, such as the SAS, are likely to obtain a good estimate of their $d_{50}$ cutoff size by use of a simplified analytical analysis if the $S / W$ ratio is kept between 1 and 5 ; this will also be the case with samplers with a high velocity and small slit width. However, in the case of a sampler with a high velocity and small slit width, a good estimate of the $d_{50}$ cut-off size is likely from the simplified analytical method when the $S / W$ ratio is higher than 5 , this being explained by the fact that the high velocity air jet from the nozzle reaches the surface in a compact form, and hence retains a similar radius of curvature to the nozzle radius.

A major problem in designing an efficient microbial air sampler is to ensure an efficient collection of smaller sizes of microbe-carrying particles on the agar surface. The two main variables that influence impact efficiency are nozzle size and intake air velocity, and these should be optimised. The lower diameter of microbe-carrying particles measured by a sampler in an occupied room is about $1 \mu \mathrm{m}$ and if the sampler should efficiently collect all sizes down to $1 \mu \mathrm{m}$, as suggested in ISO 14698-1 (2003), then a good starting point in designing an efficient microbial sampler is to use a nozzle radius, or half-slit width, of not greater than $0.5 \mathrm{~mm}$, and velocity of about 20-30 $\mathrm{m} / \mathrm{s}$. It can be readily understood that as the velocity increases, so will impaction, but a fact often overlooked is that if the nozzle is small, the radius of curvature will be small, and the impact efficiency will be high, The $S$ / $W$ ratio should also be considered and, if kept between 1 and 5, the efficiency of impaction calculated by the simplified analytical method for a sampler designed with nozzle and air velocity close to those recommended, will be reasonably accurate. It should also be noted that a sampler with a $S / W$ ratio below 1 will not be an inefficient sampler, but will simply be more efficient than that calculated by the simplified analytical method. If a more exact calculation of impact efficiency is required when the $S$ / $W$ ratio is below 1 , the CFD method should be used. Although the impact efficiency calculated by the simplified analytical method in samplers with a $S / W$ ratio above 5 may be reasonably accurately, this is best confirmed by CFD analysis

It has been demonstrated by Stewart et al. (1995) that air velocity has an effect on the growth of microbes impacted on agar, although May and Harper (1957) working with an impinger showed that high velocities had no effect on the viability of a sensitive microbe. The reason for the difference in opinion between the two authors is not clear, although it could be speculated that it may have been caused by increased dehydration when impacting onto agar rather than liquid. However, irrespective of the reason, to minimise the loss of viability, sampling should be carried out at lower rather than higher velocities, and our suggested velocity of around 20-30 m/s almost coincides with that found by Stewart et al. (1995) for the least losses.

The microbe-carrying particles in an occupied space are mostly carried by human detritus and, because of their larger size, are much easier to impact onto an agar surface than single-cell microbes. A method has been derived to ascertain the proportion of microbe-carrying particles sampled from a typical size distribution of 
microbe-carrying particles found in an occupied room. It was calculated that the samplers with a $d_{50}$ of about 0.2, 2 and $19 \mu \mathrm{m}$ would collect about $100 \%$, 98\%, and 22\%, respectively, of all of the microbe-carrying particles found in an occupied room.

To ensure a high efficiency of impaction of airborne microbes, consideration should also be made of the losses at the entrance of the intake nozzle caused by impaction of particles from the air flow onto the nozzle, or from the air vortexes induced at the intake. These will be more important in samplers with a high intake velocity. If the sampler's intake nozzle is bell shaped, or has an angled lead into the nozzle, there will be less likelihood of particles being lost than with a sharp-edged $90^{\circ}$ nozzle intake found in many microbial air samplers.

It is clear that when inefficient samplers are used in rooms, which have an airborne microbial concentration above the regulatory limit, counts may be obtained that suggest the rooms are satisfactory. It is therefore necessary that samplers should have high collection efficiency, and the methods outlined in this paper should aid in both designing samplers and validating existing designs. However, given the constraints of the simple analytical and CFD methods, it is best that the collection efficiency of samplers is confirmed experimentally using different diameters of test particles of a size similar to those found in an occupied room, The method given in ISO 146981 (2003) is a suitable method. It will also be useful to sample the natural microbial flora in the room and compare the sampling results with samplers of known high efficiency (Brachman et al., 1964; Henningson \& Ahlberg, 1994; Macher, 1997; Ljungqvist \& Reinmuller, 1998).

\section{References}

1. Brachman, P.S., Ehrlich, R., Eichenwald, H.F., Gabelli, V.J., Kethley, T.W., Madin, S.H. et al (1964). Standard sampler for assay of airborne microorganisms. Science, 144, 1295.

2. Davies, C. N., \& Aylward, M. (1951). Trajectories of heavy, solid particles in a two dimensional jet of ideal fluid impinging normally on a plate. Proceedings of the Physical Society (London), B64, 889,

3. Davies, R.R., and Noble, W.C. (1962). Dispersal of bacteria on desquamated skin. Lancet, 2, 1459.

4. Henningson, E.W., and Ahlberg, M.S. (1994). Evaluation of microbial aerosol samplers: a review. Journal of Aerosol Science, 25, 1459.

5. Hinds, W.C. (1999). Aerosol technology; properties, behaviour, and measurement of airborne particles. Wiley-Interscience, New York, USA. ISBN 0-471-19410-7.

6. ISO 14698-1:2003. Cleanliness and associated controlled environments - Biocontamination Control: Part 1: General principles and methods. Inter etc

7. Lach, V. (1985). Performance of the surface air system air samplers. Journal of Hospital Infection, 6, 102.

8. Ljungqvist, B., and Reinmüller, B. (1998). Active sampling of airborne viable particles in controlled environments: a comparative study of common instruments. European Journal of Parenteral Science, 3(3), 59.

9. Macher, J.M. 1997. Evaluation of bioaerosol performance. Applied Occupational Environmental Hygiene. 12. 730.

10. Marple, V.A., and Liu, B.Y.H. (1974). Characteristics of laminar jet impactors. Environmental Science and Technology, 8, 648.

11. Marple, V. A., \& Willeke, K. (1976). Impactor design. Atmospheric Environment, 10, 891.

12. May, K. R., \& Harper, G. J. (1957). The efficiency of various liquid impinger samplers in bacterial aerosols. British Journal of Industrial Medicine, 14, 287.

13. Möller, A.L. (2002). Measurement of airborne microorganisms - a long development still with problems. Monitor of the Scottish Society of contamination Control, 45, 2.

14. Nevalainen, A., Pastuszka, J. Liebhaber, F. \& Willeke, K. (1992). Performance of bioaerosol samplers: Collection characteristics and sampler design considerations. Atmospheric Environment, 26A, 531.

15. Noble, W.C., Lidwell, O.M., and Kingston, D. (1963).The size distribution of airborne particles carrying microorganisms. Journal of Hygiene (Cambridge), 61, 385. 
16. Ranz, W.E., and Wong, J.B. (1950). Jet impactors for determining the particle size distribution of aerosols. Archives of Industrial Hygiene and Occupational Medicine, 5, 464.

17. Shi, C.Y. and X, G. X. (2004). Skin dose depth profiles for monoenergetic point gamma sources. American Nuclear Society Transactions; 90: 505-506.

18. Stewart, S.L., Grinshpun, S.A., Willeke, K., Terzieva, S., Ulevicius, V., and Donnelly, J. (1995). Effect of impact stress on microbial recovery on an agar surface. Applied and Environmental Microbiology, $61,1232$.

19. Whyte, W. (1986). Sterility assurance and models for assessing airborne bacterial contamination. Journal of Parenteral Science and Technology, 40, 188.

20. Willeke, K. and McFeters, J.J. (1975). The influence of flow entry and collection surface on the impaction efficiency of inertial impactors. Journal of Colloid and Interface Science, 53, 121. 